\title{
Instrumentación de un impulsor para lámpara de LED
}

\section{Instrumentation of an LED Lamp Driver}

\author{
González-Ventura José Ranulfo \\ Universidad Autónoma de San Luis Potosí \\ Facultad de Ciencias, México \\ Correo:jose11jglz@gmail.com \\ Campos-Cantón Isaac \\ Universidad Autónoma de San Luis Potosí \\ Facultad de Ciencias, México \\ Correo: :icampos@fciencias.uaslp.mx
}

\author{
Camacho-Juárez Sergio \\ Universidad Autónoma de San Luis Potosí \\ Facultad de Ciencias, México \\ Correo:sergio_camacho@uaslp.mx \\ Núñez-Olvera Oscar Fernando \\ Universidad Autónoma de San Luis Potosí \\ Instituto de Investigación en Comunicación Óptica, México \\ Correo:oscar_n@cactus.iico.uaslp.mx
}

Información del artículo: recibido: abril de 2015, reevaluado: septiembre de 2015 y mayo de 2016, aceptado: julio de 2016

\section{Resumen}

En este artículo se realiza la instrumentación de un impulsor para una lámpara de LED. Se busca controlar la intensidad luminosa de la lámpara LED a través de la técnica PWM, con circuitos RC y Mosfet. Se realiza la instrumentación de la propuesta eléctrica a través del empleo del concepto de reactancia capacitiva para limitar la corriente de entrada proveniente de la línea de CA.

\section{Abstract}

This article describes the implementation of an LED lamp driver. It seeks to control the brightness of the LED lamp through PWM technique, RC and MOSFET circuits. An electrical instrumentation proposal is made by using the concept of capacitive reactance to limit the input current mains AC.

\section{Descriptores:}

- electrónica analógica

- tecnología LED

- amplificadores operacionales

- circuitos RC

- Mosfet

\section{Keywords:}

- analog electronics

- LED Technology

- operational amplifiers

- RC circuit

- Mosfet 


\section{Introducción}

El cambio climático es cada día más notorio, afecta a los ecosistemas y por ende a los seres humanos; gran parte de estos cambios observados en el clima pueden asociarse con los gases de efecto invernadero en nuestra atmósfera, ocasionados mayormente por el uso inmoderado de combustibles fósiles para la producción de energía (USEPA, 2014). Con la finalidad de evitar la contaminación del medio ambiente se emplean con mayor frecuencia las energías renovables o energías alternativas, ya que no producen gases de efecto invernadero como las energías proporcionadas por combustibles fósiles.

El inconveniente al utilizar un tipo de energía alternativa son los costos elevados o hacer una inversión inicial donde la recuperación se refleje de mediano a largo plazo. Aplicar esto a los hogares donde no se tienen contempladas inversiones costosas lo hace innecesario si se cuenta con otra forma de energía. Si no se puede contemplar un equipo para la producción de energía como un panel solar, aero-generador, etcétera.

Una forma de contribuir con el ahorro energético puede ser la utilización de dispositivos más eficientes en el consumo de energía, en este caso, nos enfocaremos al área de iluminación que es primordial para realizar las actividades cotidianas debido a la necesidad de iluminar los lugares de trabajo o descanso.

En el área de la iluminación se encuentra el uso de la lámpara incandescente, la cual está por erradicarse, ya que consume demasiada energía, es poco eficiente y ofrece poca energía lumínica; por ello se sustituye por la lámpara fluorescente compacta (LFC), la cual consume menos energía comparada con la anterior, ya que tiene mayor tiempo de horas luz, así como mayor intensidad lumínica (CFE 2014), con la desventaja de contener elementos tóxicos como el mercurio, necesario para su funcionamiento (EPA 2014).

De esta forma se buscan nuevos dispositivos más eficientes como el caso del diodo emisor de luz (LED) (ANES, 2014) que consume menos energía que las lámparas mencionadas anteriormente, tiene mayor tiempo de vida útil, mayor intensidad lumínica y es pequeño, lo que facilita el diseño para lámparas.

Como se mencionó, una forma de reducir el uso de energía específicamente aplicado al área de iluminación es el uso de tecnología LED, estos dispositivos se aplican a distintas áreas obteniendo resultados satisfactorios, por ejemplo, se observan en medicina principalmente en terapias conocidas como lumino-terapia, en faros automotrices, pantallas LED, en lámparas de mano, en lámparas para el alumbrado de zonas urba- nas, al interior del hogar y otras aplicaciones más (mediatric 2012).

Los diodos emisores de luz están libres de los peligros que genera el mercurio que contienen las lámparas fluorescentes compactas (LFC). Son más eficientes que las bombillas tradicionales y las mismas LFC. La aplicación de la tecnología LED se motiva con la finalidad de contribuir en la reducción del consumo de energía y con ello al calentamiento global, así como remediar la contaminación lumínica por ser luz direccional (CFE 2014).

En este trabajo se propone aplicar la técnica de modulación por ancho de pulso (PWM) (Chiu 2010; Ning 2013) para controlar la corriente y la intensidad de los LEDs, así prolongar el tiempo de funcionamiento y por ende la vida útil del mismo. También se incorpora un limitador de corriente a través del concepto de reactancia capacitiva, decrementando con ello los costos al no emplear convertidores cd-cd.

Como resultado, se tiene una propuesta de iluminación que contribuye con el cuidado del medio ambiente y el gasto innecesario de energía, proporcionándole al ser humano una mayor comodidad y, con ello, una mejor calidad de vida para el desarrollo de la sociedad.

En la segunda sección se analiza la forma de limitar la corriente a través de la reactancia capacitiva. La propuesta del circuito realizado se presenta en la sección 3. En el siguiente apartado se muestran los resultados experimentales y finalmente las conclusiones.

\section{Métodos}

Se analiza la opción de controlar la corriente que circula por los LED's a través de la reactancia capacitiva, por ser a nivel experimental, la mejor forma de limitar la corriente en voltaje alterno sin elevar el consumo de potencia. La etapa de rectificado es necesaria debido al proceso de transformación de corriente alterna (CA) a corriente directa $(\mathrm{CD})$ para la entrada del impulsor, como se muestra en la figura 1. Posterior a este análisis se dan las relaciones matemáticas básicas de los diferentes bloques que conforman al impulsor LED (figura 1).

De forma general el funcionamiento del impulsor LED toma como alimentación una línea de CA que se convierte a CD utilizando un rectificador, el cual suministra corriente a los LED's.

Para tener un manejo de energía eficiente se utiliza la técnica de conmutación por ancho de pulso $(\mathrm{PWM})$ y con ella se regula la intensidad de corriente que circula por los diodos LED, ya que se puede controlar el ciclo de trabajo y, a su vez, un tiempo de descanso en estado de apagado lo que prolonga la vida útil del LED. En general, si se tiene una fuente de corriente alterna de 


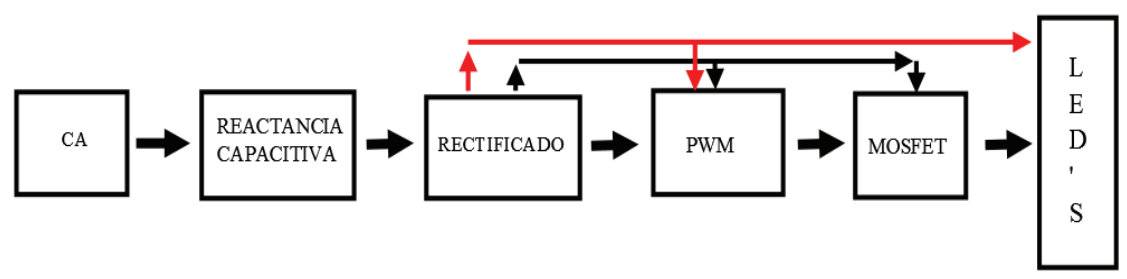

Figura 1. Diagrama a bloques de un impulsor LED

$120 \mathrm{~V}$, esta se rectificará y disminuirá su voltaje según lo requiera el arreglo de LED, posteriormente se le incorpora un PWM para la conmutación del Mosfet.

\section{Reactancia capacitiva}

El método de la reactancia capacitiva parte del concepto de potencia compleja, que en coordenadas rectangulares se escribe como

$P_{\text {compleja }}=P+j Q$

Donde P es la potencia promedio y $\mathrm{Q}$ se denomina potencia reactiva provocada por las capacitancias y bobinas del circuito. La dimensión de $\mathrm{Q}$ se define como volt-ampere-reactivo (VAR)

$Q=V_{e f} I_{e f} \operatorname{sen}(\theta-\varnothing)$

de aquí el nombre del método de reactancia capacitiva. Entonces, partiendo de la relación voltaje-corriente

$I=C S V$

se deduce la impedancia como

$Z=1 / S C$

Para ver la relación con la frecuencia se sustituye $S=j \omega$ variable en el plano complejo.

Por lo tanto

$Z=1 / j \omega C$

de donde

$X C=1 / \omega C$

que es lo que buscamos.

\section{LED}

Del bloque de LED's se observa que existen distintos fabricantes donde el brillo y corriente de consumo son diferentes, aunque a simple vista se vean iguales, sin embargo, al conectarse a una fuente de tensión con la misma cantidad de corriente, estos pueden ofrecer distinto brillo, lo que se debe tener en cuenta para el diseño de proyectos con LED's.

Para que se tenga el mismo brillo y la vida útil sea la óptima, la corriente debe apegarse al consumo proporcionado por el fabricante. La corriente y brillo del LED se pueden manejar utilizando un modulador por ancho de pulso y lograr un óptimo funcionamiento. De otra manera, la corriente estaría variando entre la corriente óptima y corriente máxima o corriente mínima, con ello cambiando su intensidad luminosa y reduciendo su vida útil.

El brillo de los LED's se garantiza perfectamente solo utilizando la corriente nominal, para este caso, 20mA (figura 2).

\section{Mosfet}

Este bloque se constituye por un Mosfet de potencia, que es un dispositivo controlado por voltaje en su compuerta. La velocidad de conmutación es muy alta, y los tiempos de conmutación son del orden de nanosegundos. Estos tienen el problema de descarga electrostática y requieren cuidados especiales en su manejo, además de ser relativamente difícil protegerlos en condiciones de falla por cortocircuito (Rashid 2004).

\section{PWM}

Existen distintas técnicas de PWM, una de ellas es la Modulación por ancho de pulso sinusoidal. Esta es una de

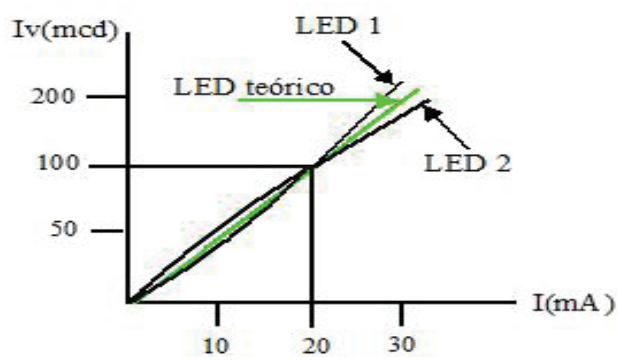

Figura 2. Gráfica de la corriente óptima de funcionamiento para cada LED con la intensidad lumínica apropiada 
las más utilizadas en la industria por su baja distorsión armónica, ya que se crea empleando una señal portadora y otra moduladora.

La construcción típica de un circuito PWM se lleva a cabo mediante un comparador con dos entradas y una salida. Una de las entradas se conecta a una onda portadora diente de sierra (triangular), mientras que la otra queda disponible para la señal moduladora sinusoidal proporcionada por un generador de ondas. La frecuencia de salida es igual a la señal del diente de sierra y el ciclo de trabajo está en función de la portadora.

$D=t / T$

donde

$\mathrm{D}=$ ciclo de trabajo

$\mathrm{t}$ = tiempo de encendido (ancho del pulso)

$\mathrm{T}$ = período del diente de sierra (figura 3 )

\section{Propuesta del circuito}

El arreglo experimental se muestra en la figura 4, este se encuentra dividido en bloques. El cuadro azul (1) es la fuente para alimentar a los LED's, el cuadro verde (2) es la fuente regulada para alimentar al PWM, el cuadro

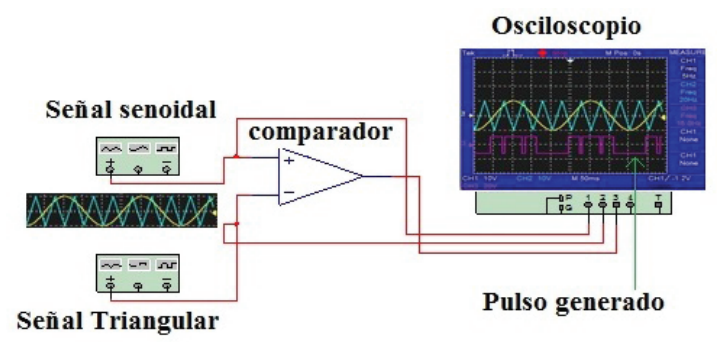

Figura 3. Ilustración de la señal PWM generada amarillo (3) muestra la fuente regulada para controlar al mosfet por medio de un opto-acoplador 4N27 el cual recibe la señal del PWM, y en el cuadro marrón (4) están los LED's en serie. Las líneas tenues restantes son v+.

Como se puede ver en la figura 4, es necesario limitar la corriente a la entrada de las dos fuentes (cuadros azul (1) y verde (2)) para ello se utilizan las fórmulas 3 y 6.

Para la fuente de voltaje en el cuadro azul (1) su consumo total de corriente es de $70 \mathrm{~mA}$ incluyendo los LED's, a un voltaje de $126 \mathrm{~V}$ (voltaje de línea) ello se encuentra con la ecuación 3, donde XC es de $1.71 \mathrm{k}$ $\mathrm{Ohm}$. La capacitancia se da a partir de la ecuación 6 donde resulta $1.5 \mathrm{uF}$.

Para la fuente del recuadro verde (2), los requerimientos son de $22 \mathrm{~mA}$ de corriente incluyendo el circuito $\mathrm{PWM}$, que se alimenta por el voltaje de línea. Con ello, XC es de $5.72 \mathrm{k}$ Ohm y una capacitancia de $46.3 \mathrm{uF}$.

Para proporcionar la función PWM se utilizó el integrado TL494 que es de bajo costo.

El opto-acoplador 4 N27 es un dispositivo que sirve para aislar la etapa de potencia de la etapa de control. El funcionamiento del opto-acoplador se da por medio de un LED y un transistor. Su funcionamiento es el siguiente (figura 5): al tener alimentación el LED se enciende y envía luz a la base del transistor, que al recibirla se coloca en modo de saturación y de esta forma puede controlar los dispositivos.

Los elementos utilizados en el arreglo experimental de la figura 4 son: entrada AC de línea $120 \mathrm{v}, \mathrm{C} 1=$ $1.5 \mathrm{uf} / 250 \mathrm{v}, \mathrm{C} 2=0.47 \mathrm{uF} / 250 \mathrm{v}, \mathrm{C} 3=0.01 \mathrm{uF}, \mathrm{C} 4=47 \mathrm{uF} / 50 \mathrm{v}$, $\mathrm{C} 5=10 \mathrm{uF} / 350 \mathrm{v}, \mathrm{C} 6=0.01 \mathrm{uf}, \mathrm{D} 1=1 \mathrm{~N} 4003, \mathrm{D} 2=1 \mathrm{~N} 4003$, $\mathrm{D} 3=$ zener 9v, D4 = 1N4003, D5 = zener 7v, D6 = diodo LED 10v, R1 $=330 \mathrm{~K} \Omega, \mathrm{R} 2=10 \Omega, \mathrm{R} 3=330 \mathrm{~K} \Omega, \mathrm{R} 4=22 \Omega$, $\mathrm{R} 5=330 \mathrm{~K} \Omega, \mathrm{R} 6=50 \Omega, \mathrm{R} 7=33 \mathrm{~K} \Omega, \mathrm{PWM}=\mathrm{TL} 494$, Optoacoplador $=4 \mathrm{~N} 25$ y BJT $=$ IRF 730 .

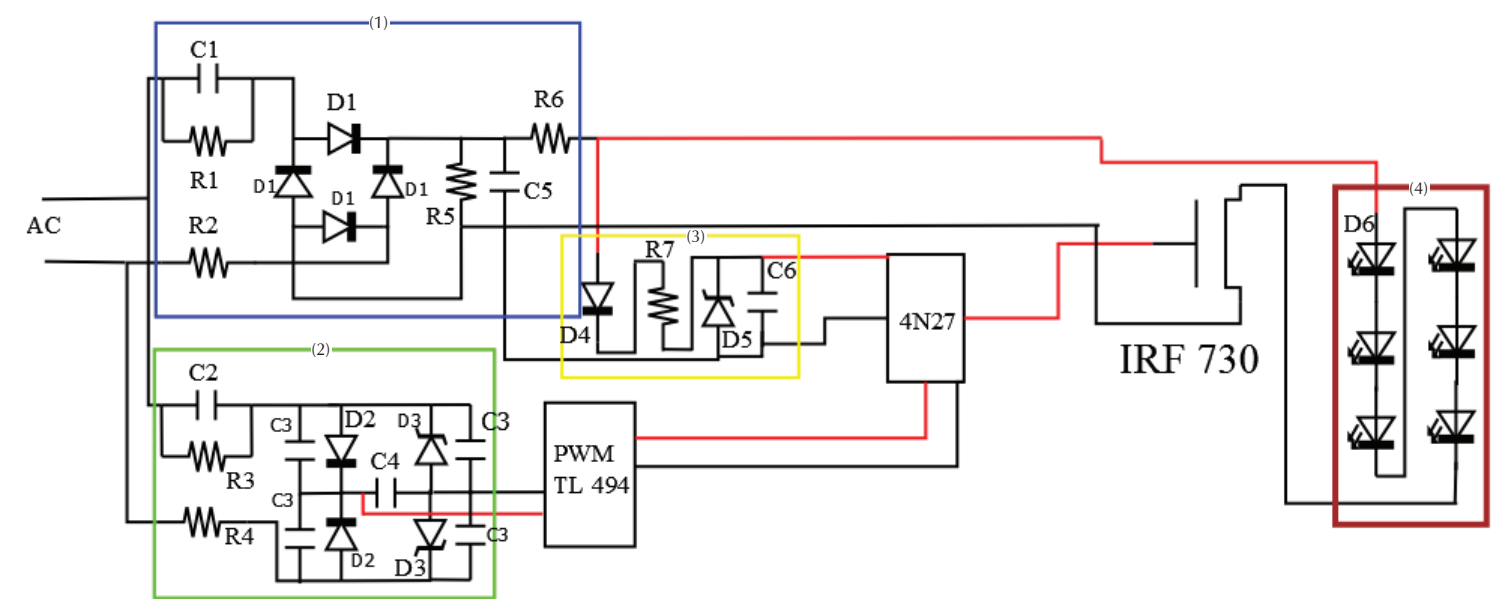

Figura 4. Arreglo experimental 


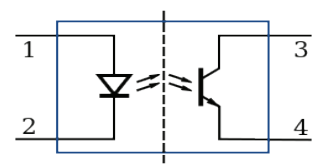

Figura 5. Símbolo eléctrico de un opto acoplador

Con referencia a la figura 1 podemos descomponer las partes que integran el circuito de la figura 4 como se muestra en la figura 6, en donde entre los bloques azul (1) y verde (2) se encuentra el limitador de corriente dentro del bloque denominado reactancia capacitiva. Para la transformación de corriente alterna a corriente directa se encuentran los puentes de diodos comprendidos en los mismos bloques azul (1) y verde (2) de la figura 4, el del bloque azul proporciona energía a los LEDs y al impulsor de estos (Mosfet), y el del bloque amarillo (3) para alimentar al PWM, estos comprendidos en el bloque denominado rectificador de la figura 6 . También encontramos el bloque PWM en ambas figuras compuesto por un integrado TL 494, el bloque denominado Mosfet (figura 6) con referencia a la figura 4 lo comprende el cuadro marcado en amarillo (3) y el IRF en esta figura; finalmente el bloque de LEDs es igual para ambas figuras.

\section{Resultados experimentales}

El desarrollo experimental se muestra en la figura 7, donde el cuadro azul (1) es la fuente para alimentar a los LED's, el cuadro verde (2) es la fuente regulada para alimentar al PWM, el cuadro amarillo (5) muestra la fuente regulada para controlar al Mosfet por medio de un opto-acoplador 4N27, el cual recibe la señal del PWM, en el cuadro marrón (6) están los 8 LED's que serán encapsulados por una bombilla, en el cuadro rojo (3) se encuentra el PWM y en el cuadro naranja (4) el Mosfet. Las líneas en rojo son v+ y en negro la tierra del circuito.

A continuación se muestran las señales generadas por cada etapa del prototipo experimental (figura 7) con el fin de corroborar su funcionamiento durante el periodo donde la señal portadora es de $2.5 \mathrm{~ms}$ (para todos los experimentos) y ciclo de trabajo de $75 \%$. La figura 8a muestra una señal sinusoidal de 120 v y $60 \mathrm{~Hz}$ aplicada a la entrada del bloque azul (1) y verde (2) (señal CA figura 6). En la figura 8b y c se observan las señales de la reactancia capacitiva que se encuentran también en los bloques azul (1) y verde (2) de la figura 7 (o salida del bloque reactancia capacitiva de la figura 6). En la figura $8 \mathrm{~d}$ y e se muestran las señales de salida

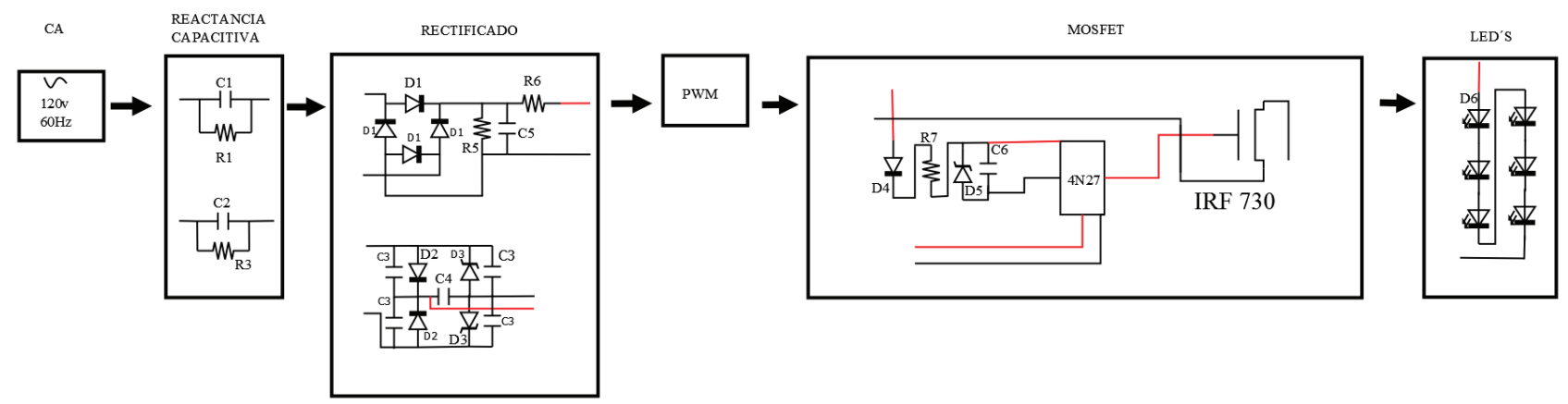

Figura 6. Diagrama a bloques del arreglo experimental esquematizado como en la figura 1

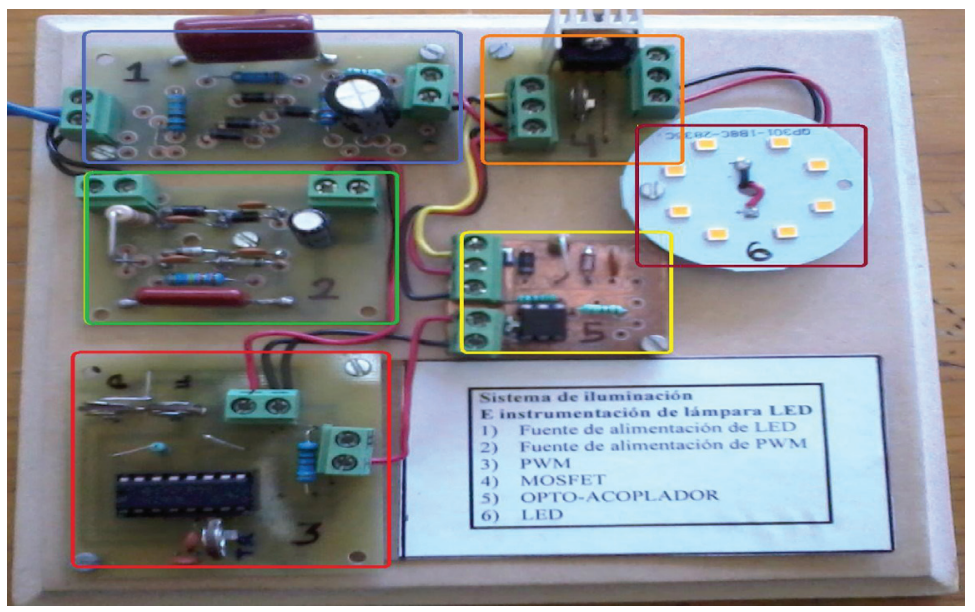

Figura 7. Prototipo experimental 
de los rectificadores de ambos bloques antes del filtro capacitivo (bloque rectificador figura 6). En la figura $8 \mathrm{f}$ se muestra la señal PWM correspondiente al cuadro en rojo (3) (bloque PWM figura 6). La señal proporcionada por el opto-acoplador comprendido en el cuadro amarillo (5) se muestra en la figura $8 \mathrm{~g}$ y la salida del cuadro naranja (4) que es el Mosfet se da en figura $8 \mathrm{~h}$ (ambos comprendidos en el bloque Mosfet de la figura 6). Finalmente, en la figura $8 \mathrm{i}$ se presenta la señal a través de los LEDs, cuadro marrón (6).

Posterior a la corroboración del funcionamiento del prototipo experimental se verifica que la modulación por ancho de pulso funcione adecuadamente, esto se hace variando el ciclo de trabajo y observando la respuesta de salida del TL494 como se aprecia en la figura 9.
En este experimento se utilizó un ciclo de trabajo de $90 \%$ en el PWM, como se aprecia en la figura 10a, en la figura $10 \mathrm{~b}$ se observa el efecto de esta señal en la conmutación del MOSFET.

Los resultados de iluminación se realizaron en el laboratorio con un medidor de intensidad lumínica de la marca LIGHT METER arrojando 123 lux a una distancia de $30 \mathrm{~cm}$ de separación entre la fuente lumínica y el sensor como se aprecia en la figura 11. También se realizaron experimentos con diversos ciclos de trabajo, dos de ellos, por ejemplo, a 75\% (figura 8f) arrojando una intensidad lumínica de 115 lux y al 50\% arrojando una intensidad lumínica de 102 lux. Cuando se coloca el ciclo de trabajo del PWM a la mitad de su operación, a simple vista la lámpara tiene una excelente apariencia luminosa, pero haciendo una comparativa con los datos

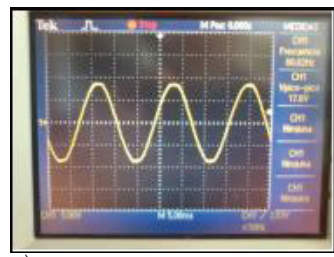

a)

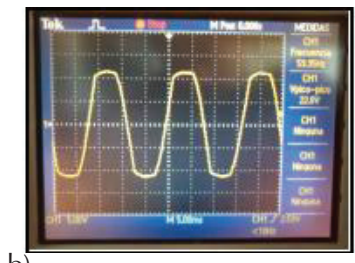

b)
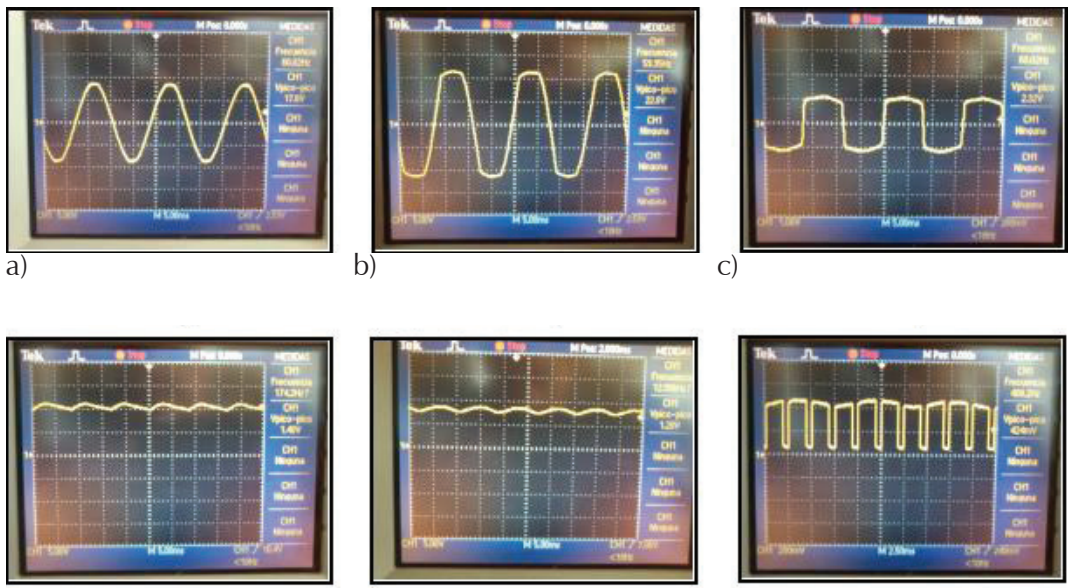

d)

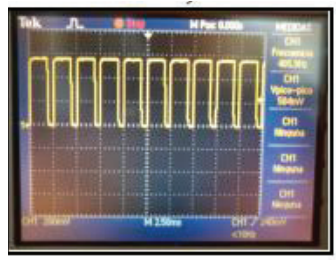

g)

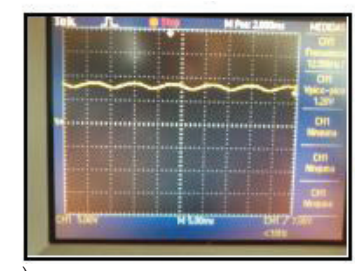

e)

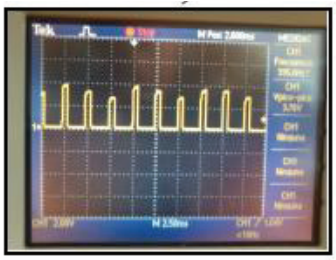

h)
C)
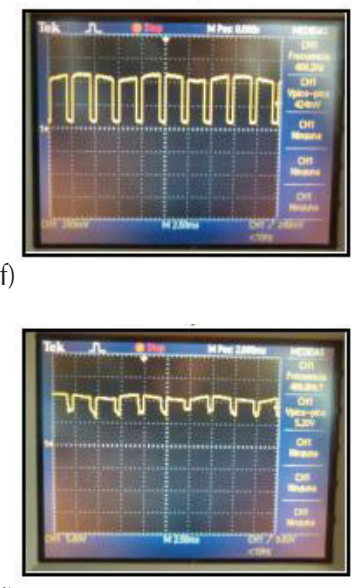

Figura 8. Señales del prototipo experimental: a) entrada al circuito, b) reactancia capacitiva de la fuente para los LEDs, c) reactancia capacitiva de la fuente PWM, d) rectificación de la fuente LEDs, e) rectificación de la fuente PWM, f) salida PWM, g) salida opto-acoplador, h) salida mosfet y i) señal troceada por los LEDs
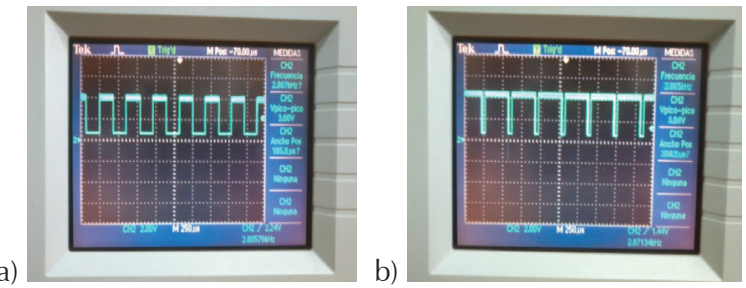

Figura 9. a) salida del PWM con un ciclo de trabajo a 50\%, b) ciclo de trabajo a $90 \%$
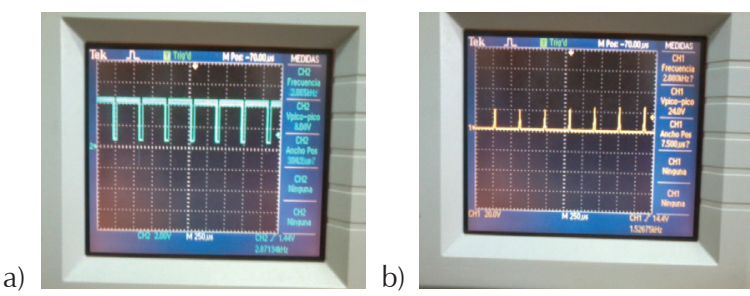

Figura 10. a) señal PWM monitoreada a 90\% del ciclo de trabajo, b) señal monitoreada a $90 \%$ a la salida de la conmutación del Mosfet 

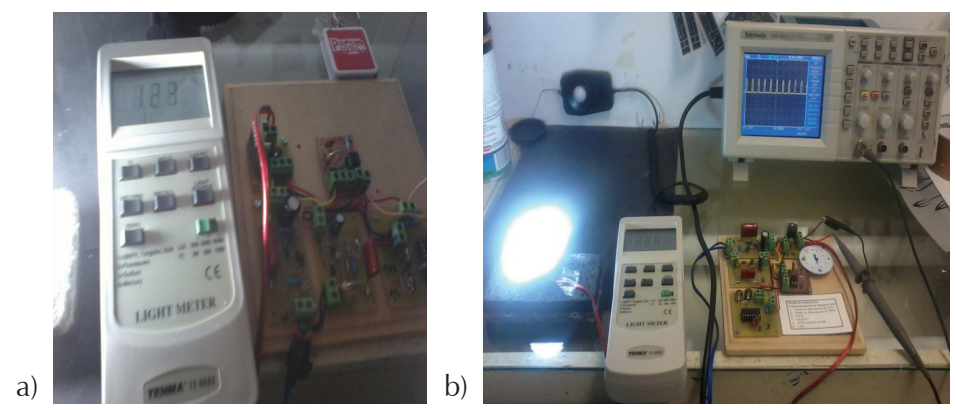

Figura 11. a) se muestra la lectura obtenida de 123 luxes a una distancia de $30 \mathrm{~cm}$ del sensor, b) lámpara encendida con un ancho de pulso del $90 \%$

del fabricante para los LEDs su punto óptimo de intensidad lumínica es de 119 lux y tiempo de vida de 20 mil horas, entonces con esta propuesta tomamos el ciclo de trabajo del PWM alrededor de 75\% alcanzando la intensidad lumínica óptima.

Estos resultados indican que la propuesta para realizar una lámpara de LED es correcta y una excelente opción para fabricar las lámparas de iluminación. Con la implementación del PWM se puede controlar la intensidad lumínica, ya que la corriente se puede variar, esto hace una buena técnica para buscar el óptimo funcionamiento de un LED por su consumo de corriente.

\section{Conclusiones}

Se puede crear una lámpara LED de bajo costo utilizando la reactancia capacitiva, esta lámpara puede ser elaborada con LED's económicos, ya que la corriente se regula de forma precisa según este trabajo.

Con este proyecto la fabricación de una lámpara LED de bajo costo resulta eficiente en la sustitución de lámparas incandescentes para las viviendas que así lo requieran, asimismo el consumo se verá reflejado en el recibo de luz. La fabricación de este tipo de lámpara puede aplicarse en lugares donde se necesite luz por horas prolongadas, pero con una ligera iluminación, ya que al necesitar una mayor intensidad el costo de fabricación lo limitarán los LED`s.

El modelo propuesto tiene la ventaja de que bajo esta técnica se pueden agregar una mayor cantidad de cadenas LED para incrementar la intensidad luminosa, sin aumentar la potencia de consumo, debido a que se colocan los LED s en serie, por lo tanto, la corriente se mantiene y se incrementa su intensidad luminosa.

Según los experimentos, la propuesta al rededor de $75 \%$ en el ciclo de trabajo del PWM es la óptima.

Como trabajo futuro se propondrá la creación de otro tipo de PWM, el cual se pueda realizar con un integrado 555 [22], y con ello llevar la lámpara de $100 \%$ a $20 \%$, de tal forma que al mover el ciclo de trabajo auto- máticamente se varíe la frecuencia. Se estudiará en laboratorio las ventajas y desventajas de dicha propuesta.

\section{Referencias}

ANES asociación nacional de energía solar [en línea] [Fecha de consulta: 15 de enero 2014].

Disponible en: www.anes.org/anes/index.php.

Chiu H.J., Lo Y.K., Chen J.T., Cheng S.J., Lin C.Y., Mou S.C. A high-efficiency dimmable LED driver for low-power lighting applications. IEEE Transactions on Industrial Electronics, volumen 57 (número 2), febrero de 2010.

CFE [en línea] [Fecha de consulta: 21 de enero 2014].

Disponible en:

www.fide.org.mx/index.php?option=com_content\&view=art icle\&id $=120 \&$ Itemid $=218$

EPA [en línea] [Fecha de consulta: 17 de enero 2014].

Disponible en:

www2.epa.gov/cfl/what-are-connections-between-mercuryand-cfls

Mediatric [en línea] [Fecha de consulta: 21 de enero 2014]. Disponible en:

mediatric.wordpress.com/2012/10/18/luminoterapia/

Ning N., Chen W.B., Yu D.J., Feng C.Y., Wang C.B. Self-adaptive load technology for multiple string LED drivers. Electronics Letters, volumen 49, (número 18), agosto de 2013.

Rashid M.H. Electrónica de potencia, circuitos, dispositivos y aplicaciones, $3^{\text {a }}$ ed., editorial Peason Prentice Hall, 2004.

USEPA (United States Environmental Protection Agency) [en línea] [Fecha de consulta: 15 de enero 2014]. Disponible en: www.epa.gov/espanol/cambioclimatico/ciencia/indicadores. html 


\section{Este artículo se cita: \\ Citación estilo Chicago \\ González-Ventura, José Ranulfo, Isaac Campos-Cantón, Sergio Camacho-Juárez, Oscar Fernando Núñez-Olvera. Instrumenta- ción de un impulsor para lámpara de LED. Ingeniería Investigación y Tecnología, XVII, 04 (2016): 445-452.}

\section{Citación estilo ISO 690}

González-Ventura J.R., Campos-Cantón I., Camacho-Juárez S., Núñez-Olvera O.F. Instrumentación de un impulsor para lámpara de LED. Ingeniería Investigación y Tecnología, volumen XVII (número 4), octubre-diciembre 2016: 445-452.

\section{Semblanzas de los autores}

José Ranulfo González-Ventura. Se tituló como licenciado en electrónica en diciembre del 2015 por la Facultad de Ciencias, UASLP. Su área de interés es la instrumentación de circuitos electrónicos, energías renovables.

Isaac Campos-Cantón. Recibió el grado de maestro en ingeniería en 1997 por la Facultad de Ingeniería de la UNAM y el grado de doctor en 2009 por el IICO-UASLP. Actualmente es profesor de tiempo completo en el área de electrónica de la Facultad de Ciencias, UASLP. Sus áreas de interés son la instrumentación y desarrollo de circuitos electrónicos lineales y no lineales.

Sergio Camacho-Juárez. Licenciado en ingeniería biomédica en 2000 y maestro en ciencias en ingenierías biomédicas en 2002 por la Universidad Autónoma Metropolitana. Obtuvo el doctorado en ciencias biomédicas y biotecnología molecular en el 2012 por el IPN, México. Actualmente es profesor en la Facultad de Ciencias, UASLP. Sus áreas de interés son aplicaciones biomédicas.

Oscar Fernando Núñez-Olvera. Es licenciado en ingeniería industrial área electrónica (1985) por el Instituto Tecnológico de San Luis Potosí, asimismo obtuvo el grado de maestro en ciencias en ingeniería electrónica en 1991 por el Centro Nacional de Investigación y Desarrollo Tecnológico y el grado de doctor en ingeniería eléctrica en 2012 por la UASLP. Actualmente es profesor en el Instituto de Investigación en Comunicación Óptica UASLP. Sus áreas de interés son instrumentación e interfaces electrónicas. 